\title{
Molecular Characterization and Analysis of the acrB Gene of Aspergillus nidulans: A Gene Identified by Genetic Interaction As a Component of the Regulatory Network That Includes the CreB Deubiquitination Enzyme
}

\author{
Natasha A. Boase, Robin A. Lockington, Julian R. J. Adams, Louise Rodbourn \\ and Joan M. Kelly ${ }^{1}$
}

School of Molecular and Biomedical Science, University of Adelaide, Adelaide, 5005, SA, Australia

Manuscript received October 31, 2002

Accepted for publication January 27, 2003

\begin{abstract}
Mutations in the $a c r B$ gene, which were originally selected through their resistance to acriflavine, also result in reduced growth on a range of sole carbon sources, including fructose, cellobiose, raffinose, and starch, and reduced utilization of $\omega$-amino acids, including GABA and $\beta$-alanine, as sole carbon and nitrogen sources. The $a c r B 2$ mutation suppresses the phenotypic effects of mutations in the $c r e B$ gene that encodes a regulatory deubiquitinating enzyme, and in the $\mathrm{cre} C$ gene that encodes a WD40-repeat-containing protein. Thus AcrB interacts with a regulatory network controlling carbon source utilization that involves ubiquitination and deubiquitination. The $a c r B$ gene was cloned and physically analyzed, and it encodes a novel protein that contains three putative transmembrane domains and a coiled-coil region. AcrB may play a role in the ubiquitination aspect of this regulatory network.
\end{abstract}

$\mathrm{T}$ HE acrB2 mutation was isolated in the filamentous fungus Aspergillus nidulans as a spontaneous resistant sector on complete medium containing acriflavine in a genetic screen with the joint aims of understanding acriflavine toxicity and obtaining extra tools for genetic mapping (Roper and KAFER 1957). A strain containing the $a c r B 2$ mutation also showed greater resistance to the presence of crystal violet and malachite green than that showed by the wild-type strain, and the acrB2 mutant allele is recessive to the wild-type allele (ROPER and KAFEr 1957; Arst 1981). Like acrB mutations, the creD34 mutation also confers resistance to acriflavine (KeLLY and Hynes 1977; Arst 1981). The creD34 mutation was identified as a suppressor of the effects of the creC27 mutation, and it was also shown to suppress the effects of the creB15 mutation (Kelly and Hynes 1977). Mutations in $\mathrm{creB}$ and $\mathrm{cre} C$ result in altered regulation of carbon metabolism. The $\mathrm{creB}$ gene encodes a functional deubiquitinating enzyme (Lockington and Kelly 2001) and the creC gene encodes a protein that contains five WD40-repeat motifs and a proline-rich region (Topd et al. 2000). CreB and CreC have been shown to be present in a complex in vivo (Lockington and Kelly 2002), and thus the CreB/CreC complex is part of the deubiquitination aspect of a regulatory ubiquitination

Sequence data from this article have been deposited with the EMBL/GenBank Data Libraries under accession no. AF485329.

${ }^{1}$ Corresponding author: School of Molecular and Biomedical Science, Molecular Life Sciences Building, University of Adelaide, North Terrace, Adelaide, 5005, SA, Australia.

E-mail: joan.kelly@adelaide.edu.au pathway that is required for correctly regulated carbon metabolism.

Protein stability is a key regulatory mechanism in the control of metabolism, the cell cycle, cell growth and development, and disease. Selective degradation or stabilization of intracellular proteins by ubiquitin-dependent pathways is essential for correct regulation of many cellular processes. Ubiquitin is covalently attached to substrate proteins by a protein complex usually including an activating enzyme, a conjugating enzyme, and a protein ligase. A number of conjugating enzymes and protein ligases are in the cell, and various combinations confer substrate specificity on the system. The addition of four or more ubiquitins targets the substrates for destruction via the $26 \mathrm{~S}$ proteasome, and free ubiquitin is recycled. The addition of fewer ubiquitins can alter function or target the protein to the endosomes. This process can be opposed by the action of deubiquitinating enzymes, which remove ubiquitin from specific substrates, thus stabilizing them. Perhaps the best-studied example of a regulatory deubiquitinating enzyme in a multicellular eukaryote is the Drosophila melanogaster Fat facets deubiquitinating enzyme, which is required for correct eye development (Wu et al. 1999). Genetic analysis has revealed that the critical substrate of Fat facets in the eye is Liquid facets (CADAvid et al. 2000; CHeN and Fischer 2000; CHen et al. 2002). Liquid facets is related to mouse epsin (CHEN et al. 2002), which acts as a cargo-selective endocytic clathrin adaptor protein, which regulates clathrin-coat assembly (OLDHAM et al. 2002).

Since the role in the cell of the CreB/CreC complex is to remove ubiquitin from specific substrates, then 
TABLE 1

Aspergillus nidulans strains

\begin{tabular}{|c|c|c|}
\hline Strain & Genotype & Origin \\
\hline wt & biAl; riboB2 & Derived from Pateman et al. (1967) \\
\hline creB1937 & yA1 pabA1; creB1937; riboB2 & LOCKINGTON and Kelly (2001) \\
\hline $\operatorname{creC} 27$ & biA1; creC27 & Hynes and Kelly (1977) \\
\hline $\mathrm{creD} 34$ & creD34; riboB2 & Derived from Kelly and Hynes (1977) \\
\hline $\operatorname{acr} B 2 Y$ & $y A 2 ; \operatorname{acrB2}$; $\operatorname{choA} 1$ & FGSC869 \\
\hline acrB2G & biA $1 ;$ acrB2; riboB2 & Derived from acrB2Y \\
\hline acrB14 & pabaA1; acrB14; pantoB100 & Supplied by H. N. Arst \\
\hline $\operatorname{acrB15}$ & pabaA $1 ; \operatorname{acrB15}$ & Supplied by H. N. Arst \\
\hline $\mathrm{creB} 15 \mathrm{creD} 34$ & creB15 creD34; riboB2 niiA4 & Derived from Kelly and Hynes (1977) \\
\hline $\mathrm{creC} 27 \mathrm{creD} 34$ & creC27 creD34; riboB2 niiA4 & Derived from Kelly and Hynes (1977) \\
\hline creB1937 acrB2 & $y A^{-}$pabaA1; creB1937 acrB2 & This work \\
\hline creC27 acrB2 & $y A 2 ; \operatorname{cre} C 27$ acrB2; choA 1 & This work \\
\hline creD34 acrB2 & creD34 acrB2; niiA4 & This work \\
\hline
\end{tabular}

Gene symbols are described in Clutterbuck (1993, 1997). FGSC, Fungal Genetics Stock Center.

suppressors of the phenotypic effects of mutations in creB or cre $C$ may define components of the specific regulatory ubiquitination system for these substrates. The phenotypic similarities between acrB2 and $c r e D 34$ led us to analyze whether the $a c r B 2$ mutation is also a suppressor of the phenotypes due to $\mathrm{creB}$ and $\mathrm{cre} C$ mutations, which would indicate a role for AcrB in a regulatory ubiquitination pathway that is required for correct regulation of carbon metabolism. Here we demonstrate that mutations in $\mathrm{acrB}$ result in altered utilization of various carbon sources in $A$. nidulans. In addition, we show that the effects of the acrB2 mutation are epistatic to those due to the $c r e B$ and $c r e C$ mutations, and thus the $a c r B$ gene product is likely to play a role in the regulatory ubiquitination/deubiquitination network that includes the CreB/CreC complex. Thus, to further understand the role of AcrB, we have cloned and physically analyzed the $a c r B$ gene.

\section{MATERIALS AND METHODS}

A. nidulans strains, media, growth conditions, and manipulations: The Aspergillus media and growth conditions were as described by Cove (1966). Nitrogen sources were added at a final concentration of $10 \mathrm{~mm}$ and carbon sources at $1 \%(\mathrm{w} / \mathrm{v})$ except where otherwise indicated. Genetic manipulations were carried out using techniques described by CLUTTERBUCK (1974). The A. nidulans strains used in this study are shown in Table 1. Gene symbols are as described in Clutterbuck (1993, 1997). The $a c r B$ mutant strains were selected as spontaneous resistant sectors in the presence of acriflavine (ROPER and KAFER 1957). A strain containing the acrB2 mutation was obtained from the Fungal Genetics Stock Center, and strains containing the acrB14 and acrB15 mutations were obtained from H. N. Arst.

Molecular methods: Standard molecular techniques were as outlined in SAMBrook et al. (1989). A. nidulans genomic DNA was prepared according to LEE and TAYLOR (1990). The A. nidulans cosmid library used is described in BRODY et al. (1991).

Plasmid c24F6-14 was generated by a partial EcoRI digestion of cosmid SW23H06 followed by ligation to recircularize the products of digestion. $\mathrm{Cla}$ I subclones from c24F6-14 [p4AcrB (6-kb insert), p7AcrB (10-kb insert), and p10AcrB (2-kb insert)] were generated in the $\mathrm{pBCSK}^{+}$-based plasmid pCB1004 (CARroll et al. 1994). p4AcrBSacI $\Delta$ is a SacI-generated deletion of p4AcrB. p4AcrBKpnI $\Delta$ is a KpnI-generated deletion of $\mathrm{p} 4 \mathrm{AcrB}$.

A. nidulans was transformed according to the method of Tilburn et al. (1983). Transformants from cotransformation experiments were selected using the $r i b o B^{+}$selectable marker plasmid pPL3 (OAKLEY et al. 1987) on media lacking riboflavin. Complementation of the acrB2 mutant phenotype was scored by morphology on complete medium and resistance to the presence of $0.001 \%$ acriflavine added to complete media.

The $a c r B$ gene was sequenced using a combination of custom-designed oligonucleotide primers. Double-stranded DNA template was prepared using the Promega Wizard + SV miniprep system following the manufacturer's instructions. Sequencing reactions were as outlined in the Applied Biosystems (Foster City, CA) dye terminator system instructions and were analyzed at the Institute of Medical and Veterinary Science sequencing facility (Adelaide, Australia). Sequence analysis was performed using the Australian National Genomic Information Service facility or BioNavigator.

Intron positions were determined by the PCR amplification of $a c r B$ cDNA segments from DNA prepared from an $A$. nidulans cDNA library (kindly provided by S. Osmani) using custom oligonucleotides as primers and standard PCR conditions and cycle parameters. These products were purified and directly sequenced using the same primers used for amplification. The primer pairs used were acrB1 (5'-AGTCTTGGCTG CTATCCG-3'; -58 to -40$)$ and KBF ( ${ }^{\prime}$-GACACGCCACCA TTCGTC- $3^{\prime}$; +329 to +312$)$, KBB (5'-GCGAAGGGAAATG CAGAC- $3^{\prime} ;+280$ to +297$)$ and KB (5'-ATGGGCTAAATCGA GAGC-3'; +726 to +709$)$, AK (5'-GCAATGGATGGTTTC TGC- $3^{\prime} ;+646$ to +663$)$ and SF (5'-CTCGCCTCGCCAATA GAT-3'; + 1617 to +1600$)$, KF (5'-CGCGTGGATACAGAA GCG-3'; +1066 to +1083$)$ and AS (5'-CAATCTCTGCCTTG GCGG-3'; + 2194 to +2177), SB (5'-AAGAGGCTACCGC CGCTC-3'; + 2057 to +2074$)$ and SBB (5'-GCGAGCGGTGG CTTGGCA-3'; + 2641 to +2624 ), and SBF (5'-CTTGGGCA CATTGGCGTC-3'; + 2601 to +2618 ) and acrB5 (5'-AACCAG TCACTACACGGA-3'; +3249 to +3232$)$.

The transcription start point was determined by direct sequencing of the nested PCR product and five subcloned prod- 
wild type creD34 acrB2G acrB14 acrB15

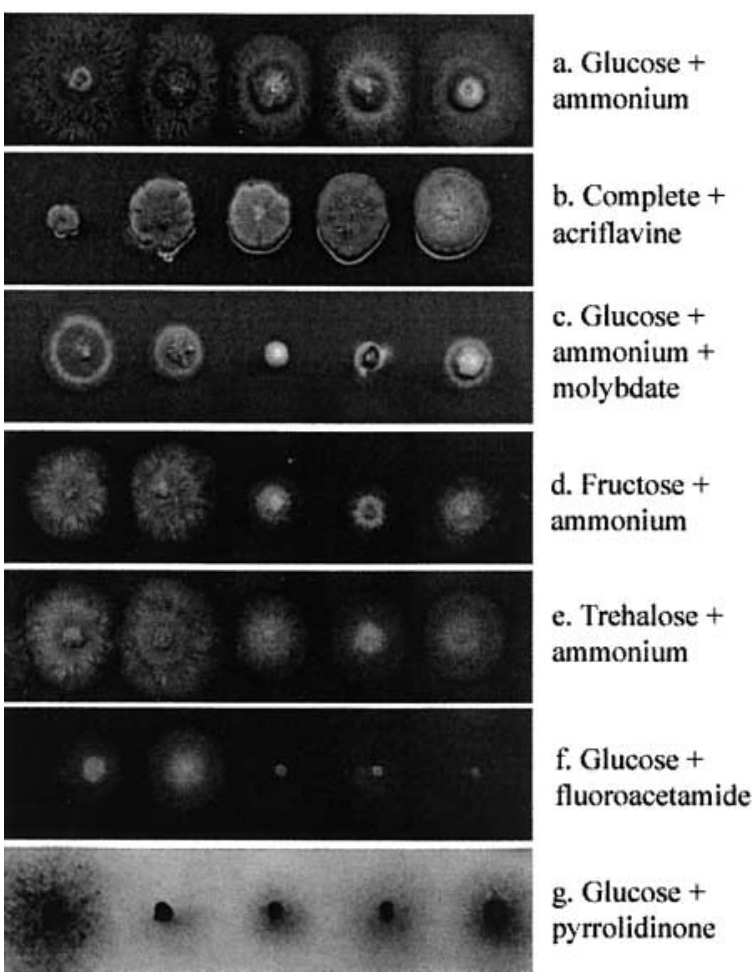

Figure 1.-Phenotypic heterogeneity among strains bearing $a c r B$ mutant alleles. Strains containing $a c r B$ mutant alleles, acrB2 (strain $a c r B 2 G$ ), acrB14, and $\operatorname{acrB15}$, were grown at $37^{\circ}$ for 2-3 days, along with a strain containing creD34 and a wildtype strain, on the following media: (a) $1 \%$ D-glucose plus 10 mu ammonium L-tartrate, (b) complete medium plus $0.001 \%$ acriflavine, (c) $1 \%$ D-glucose plus $10 \mathrm{~mm}$ ammonium L-tartrate plus $11 \mathrm{~mm}$ molybdate, (d) $1 \%$ D-fructose plus $10 \mathrm{~mm}$ ammonium L-tartrate, (e) $1 \%$ D-trehalose plus $10 \mathrm{~mm}$ ammonium L-tartrate, (f) $1 \%$ D-glucose plus $10 \mathrm{mg} / \mathrm{ml}$ fluoroacetamide, and (g) $1 \%$ D-glucose plus $10 \mathrm{~mm}$ 2-pyrrolidinone.

ucts of $5^{\prime}$ rapid amplification of cDNA ends ( $5^{\prime}$ RACE) performed on total RNA from D-glucose-grown mycelium. 5' RACE was performed using the Ambion (Austin, TX) First Choice RLM-RACE kit, following the manufacturer's instructions.

Sequences of mutant alleles were determined by direct sequencing of PCR products derived from genomic DNA of the relevant mutant strain. These were sequenced using the same procedures as for intron determination. Direct sequencing of acrB PCR products from wild-type DNA was undertaken for comparison.

\section{RESULTS}

Phenotypic analysis of $a c r B 2$, $a c r B 14$, and $a c r B 15$ : As selected, the three $a c r B$ mutant strains, acrB2, acrB14, and $a c r B 15$, were resistant to the presence of acriflavine in complete medium, with similar levels of resistance between $a c r B$ mutant strains and the $\mathrm{creD} 34$ strain (Figure 1). Since the $\mathrm{creD} 34$ mutation leads to sensitivity to molybdate, the three $a c r B$ mutant strains were tested and found to be more sensitive than the wild-type strain to the presence of $11 \mathrm{~mm}$ molybdate in synthetic complete medium, but the alleles were heterogeneous, with the $\operatorname{arr} B 2$ strain being the most sensitive to the presence of molybdate and the acrB15 strain showing lesser sensitivity. Thus the effects of the $a c r B$ mutant alleles when the toxic compounds acriflavine and molybdate are added to the medium are the same as those for creD34 and the opposite to the effects of $\mathrm{creB}$ and $\mathrm{cre} C$ mutant alleles, which confer increased sensitivity to acriflavine and resistance to molybdate added to complete medium (Hynes and Kelly 1977; Kelly and Hynes 1977; Arst 1981).

When tested for their ability to utilize various carbon sources, $a c r B$ mutant strains were found to have pleiotropic phenotypes with respect to carbon source utilization. They show decreased ability to utilize a number of different sugars as sole carbon sources in comparison to both the wild type and the creD34 mutant strain, including fructose, cellobiose, raffinose, and starch (Figure 1; Table 2). In general, the acrB14 strain showed a slightly more extreme phenotype than that showed by the acrB2 and acrB15 strains. The reduced growth on various sugars may indicate a failure of uptake or a failure to express genes encoding enzymes required for their utilization. The ability to utilize other alternate carbon sources such as quinate and glycerol was only slightly affected by the acrB mutations, with the exception of ethanol where the $a c r B$ mutants grew considerably less well than wild type (Table 2).

Test medium containing $10 \mathrm{~mm}$ allyl alcohol in the presence of glucose was used to investigate the expression of the alcohol dehydrogenase encoded by alcA. All the acrB-containing strains, like the wild-type and creD34containing strains, were resistant, indicating that they showed correct glucose repression of alcA. The $a c r B$ mutant strains were hypersensitive on medium containing glucose plus $10 \mathrm{mg} / \mathrm{ml}$ fluoroacetamide, indicating an increased level of the acetamidase expressed from the amd $S$ gene compared to the wild-type strain, which is the opposite of the phenotype conferred by the creD34 mutation. The lack of growth of the $a c r B$ mutant strains on medium containing glucose plus 10 $\mathrm{mm}$ acrylamide as a nitrogen source indicates that this increased level of amdS expression is not due to constitutive expression, as acrylamide is a substrate of the acetamidase but not an inducer of amdS (Hynes and PATEMAN 1970). Further, wild-type growth of the $a c r B$ mutant strains on acetamide as a carbon, nitrogen, or carbon and nitrogen source indicates that the $a m d S$ gene is fully inducible. Thus there is a defect in the glucose-mediated repression of amdS but no other effect on expression.

Growth on media containing the $\omega$-amino acids 10 mм $\beta$-alanine, $10 \mathrm{~mm}$ GABA, or $10 \mathrm{~mm}$ pyrrolidinone as nitrogen sources was significantly decreased in the acrB mutant strains compared to the wild-type strain, as was the case for a strain containing creD34. Similarly, growth of the mutant $a c r B$ strains on media containing 
TABLE 2

Phenotypic analysis of $a c r B$ mutant strains

\begin{tabular}{|c|c|c|c|c|c|}
\hline Growth condition & Wild type & creD34 & acrB2G & acrB14 & acrB15 \\
\hline Complete & 10 & 9 & 9 & 9 & 9 \\
\hline Glucose & 10 & 10 & 9 & 9 & 9 \\
\hline Cellobiose & 10 & 10 & 5 & 5 & 5 \\
\hline Fructose & 10 & 10 & 4 & 3 & 4 \\
\hline Galactose & 8 & 8 & 4 & 3 & 4 \\
\hline Lactose & 8 & 8 & 4 & 4 & 4 \\
\hline Maltose & 10 & 10 & 6 & 5 & 6 \\
\hline Raffinose & 10 & 10 & 6 & 6 & 6 \\
\hline Starch & 10 & 10 & 8 & 8 & 8 \\
\hline Sucrose & 10 & 10 & 7 & 7 & 7 \\
\hline Trehalose & 10 & 10 & 6 & 5 & 6 \\
\hline Xylose & 10 & 10 & 7 & 7 & 7 \\
\hline Acetate & 9 & 10 & 6 & 5 & 8 \\
\hline Ethanol & 10 & 10 & 5 & 4 & 5 \\
\hline Glucuronate & 8 & 8 & 7 & 7 & 7 \\
\hline Glycerol & 10 & 10 & 9 & 9 & 9 \\
\hline Succinate & 3 & 3 & 3 & 3 & 2 \\
\hline Quinate & 10 & 10 & 9 & 9 & 9 \\
\hline Acriflavine & 1 & 9 & 9 & 10 & 10 \\
\hline Molybdate & 10 & 5 & 2 & 3 & 4 \\
\hline Fluoroacetamide & 4 & 5 & 0 & 0 & 0 \\
\hline $2.5 \mathrm{~mm}$ allyl alcohol & 10 & 10 & 10 & 10 & 10 \\
\hline $10 \mathrm{~mm}$ allyl alcohol & 9 & 9 & 9 & 9 & 9 \\
\hline$\beta$-Alanine & 2 & 1 & 2 & 1 & 2 \\
\hline GABA & 8 & 8 & 2 & 2 & 2 \\
\hline Pyrrolidinone & 8 & 8 & 2 & 3 & 1 \\
\hline Glutamate & 8 & 8 & 4 & 4 & 4 \\
\hline Proline & 8 & 8 & 4 & 4 & 5 \\
\hline Threonine & 2 & 2 & 2 & 2 & 2 \\
\hline$\beta$-Alanine + glucose & 10 & 5 & 6 & 6 & 6 \\
\hline GABA + glucose & 10 & 8 & 5 & 5 & 5 \\
\hline Pyrrolidinone + glucose & 10 & 1 & 3 & 5 & 7 \\
\hline Glutamate + glucose & 10 & 10 & 9 & 9 & 9 \\
\hline Proline + glucose & 10 & 10 & 10 & 10 & 10 \\
\hline Threonine + glucose & 10 & 4 & 10 & 7 & 10 \\
\hline Glucose + acetamide & 10 & 9 & 10 & 9 & 10 \\
\hline Acetamide & 5 & 5 & 4 & 4 & 4 \\
\hline Acetamide $+\left[\mathrm{NH}_{4}\right]_{2} \mathrm{~T}$ & 5 & 5 & 4 & 4 & 4 \\
\hline Glucose + acrylamide & 1 & 1 & 1 & 1 & 1 \\
\hline
\end{tabular}

For full genotypes, see Table 1 . Plates were incubated at $37^{\circ}$ for 2 or 3 days. Carbon sources were added to carbon-free medium containing $10 \mathrm{~mm}$ ammonium L-tartrate at $1.2 \%$ (acetate), $1 \%$ (D-cellobiose, D-fructose, D-galactose, D-glucose, glycerol, lactose, D-maltose, D-raffinose, starch, succinate, sucrose, D-trehalose, and D-xylose), $0.5 \%$ (D-glucuronate and ethanol) or $50 \mathrm{~mm}$ [(D)-quinate]. Molybdate was added to synthetic complete medium at $11 \mathrm{~mm}$. Fluoroacetamide was added to $1 \% \mathrm{D}$-glucose medium at $10 \mathrm{mg} / \mathrm{ml}$. Allyl alcohol was added to synthetic complete medium at 2.5 or $10 \mathrm{~mm}$. Acetamide, $\beta$-alanine, GABA $(\gamma$-amino-n-butyric acid), L-glutamate, L-proline, 2-pyrrolidinone, and L-threonine were added at $10 \mathrm{~mm}$ to $1 \%$ glucose medium and at $50 \mathrm{~mm}$ when added to medium without another carbon source. Ammonium L-tartrate and acrylamide were added at $10 \mathrm{~mm}$. The overall score assigned to each colony was derived from the colony size, the hyphal density, and the amount of conidiation. For each medium, a score was assigned to define the wild-type growth, and the mutant strains were scored relative to the wild-type score. Scores are not comparable between media. Each plate test was performed on at least three separate occasions.

$50 \mathrm{~mm}$ GABA and $50 \mathrm{~mm}$ pyrrolidinone acting as both a carbon and a nitrogen source was also decreased compared to wild type and the creD34 mutant strain, but this effect was not obvious on media containing $50 \mathrm{~mm}$ $\beta$-alanine. The $a c r B$ mutant alleles affected growth to various degrees on media that had 10 mm pyrrolidinone as a nitrogen source, with the acrB15 strain having strong, almost wild-type-like growth, compared to the intermediate growth of the acrB14 strain and the weaker growth of the acrB2 strain, and this heterogeneity between alleles indicates that they do not all represent complete loss-of-function alleles. 
Growth on media containing other amino acids as nitrogen sources, such as $10 \mathrm{~mm}$ proline and $10 \mathrm{~mm}$ glutamate, was unaffected by the $a c r B$ mutations. However, decreased utilization of $50 \mathrm{~mm}$ proline and $50 \mathrm{~mm}$ glutamate when they were used as both carbon and nitrogen sources was observed for the acrB mutant strains in comparison to the wild-type strain.

Partial suppression by $a c r B 2$ of $\operatorname{creB}$ and $\operatorname{cre} C$ mutant phenotypes: The creD34 mutation was identified as a suppressor of the hypersensitivity to fluoroacetamide conferred by the creC27 mutation. The effects of the creD34 mutation are pleiotropic in that it also leads to suppression of the effects of creC27 on other enzymes subject to carbon catabolite repression, such as alcohol dehydrogenase I, but it does not suppress the effects of creC27 that are apparent under derepressing conditions, such as the poor growth on D-quinate medium (Kelly and Hynes 1977; Kelly 1980). The creD34 mutation suppresses not only some of the phenotypes conferred by the $\mathrm{cre} C 27$ mutation, but also some of the phenotypic effects of the creB15 mutation, and, weakly, of the creA204 mutation (Kelly and Hynes 1977; Kelly 1980). Similarities between the phenotypes of $c r e D$ and acr $B$ mutant strains led us to test whether the effects of mutation in $a c r B$ are also epistatic for some of the phenotypes conferred by $\mathrm{cre} B$ and $\mathrm{cre} C$ mutations.

The presence of the acrB2 mutation reversed the sensitivity conferred by the $\mathrm{creB} 1937$ and $\mathrm{creC} 27$ mutations to the presence of acriflavine in complete media (Figure 2c). The creD34 acrB2 double mutant strain was even more resistant to the presence of acriflavine than was either of the individual mutant strains, indicating an additive effect. The resistance of the creB1937 and creC27 strains to the presence of molybdate was also reversed in the relevant $a c r B 2$ double mutant strains and to a greater degree than that in the creD34 double mutant strains (Figure 2e). The acrB2 mutation also reversed the toxic effects of allyl alcohol added to D-glucose medium in creC27 and creB1937 backgrounds, the same phenotypic suppression previously seen for the creD34 mutation (Figure 2f).

The acrB2 mutation results in reduced growth compared to wild type on sole carbon sources such as fructose, maltose, starch, cellobiose, ethanol, and acetate, a phenotype not seen for the $\mathrm{creD} 34$ mutation (Figure 1; Table 2). This reduced growth is also seen in the acrB2creB1937 and acrB2creC27 double mutant strains compared to the single creB1937 and creC27 mutant phenotypes, respectively (Figure $2 \mathrm{~g}$ ). The inability of the $\operatorname{creB1937}$ and creC27 mutant strains to utilize D-quinate as an alternate carbon source was partially suppressed by the acrB2 mutation in the double mutant strains, and this effect was more pronounced than that in the $\mathrm{creD} 34$ double mutant strains (Figure $2 \mathrm{~h}$ ). Finally, the $a c r B 2 c r e B 1937$ and $a c r B 2 c r e C 27$ strains grew as poorly as the $a c r B 2$ strain on glucose medium containing 10 mm pyrrolidinone as the nitrogen source (Figure 2i).

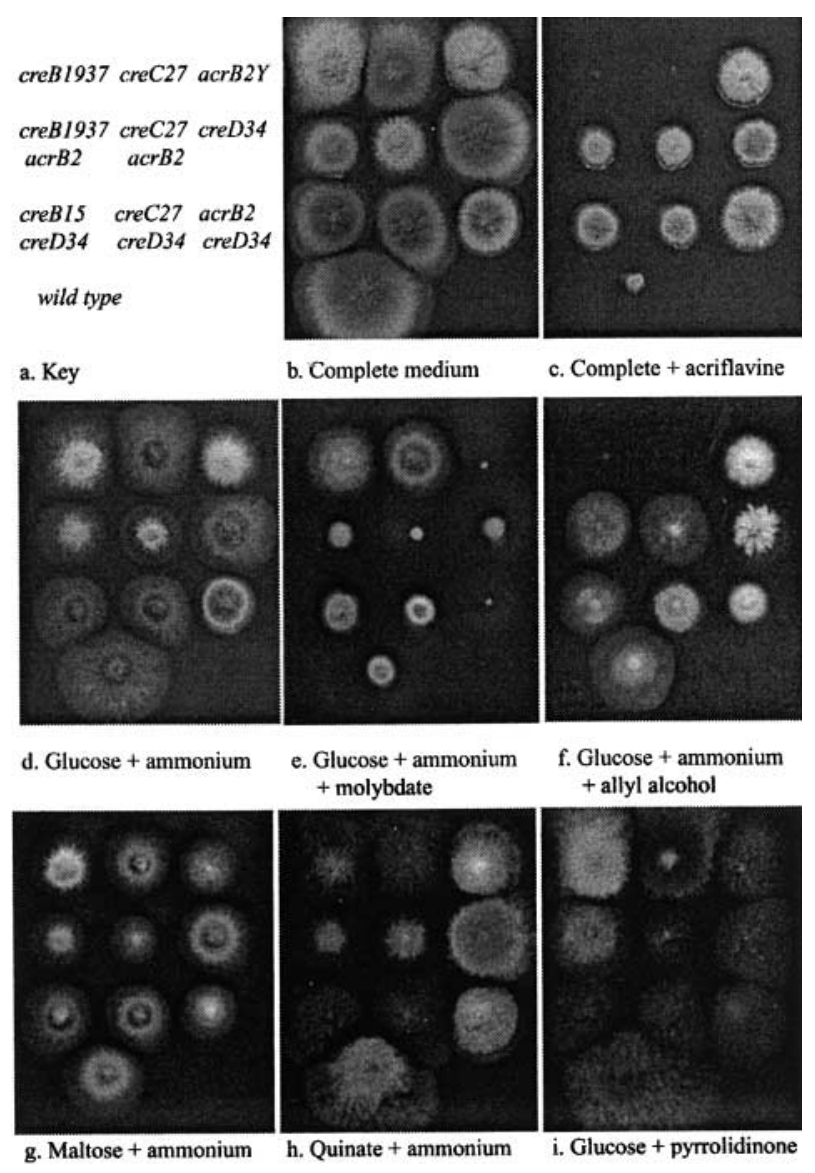

Figure 2.-Epistatic effects of the acrB2 mutation on $\mathrm{creB}$ and cre $C$ mutant phenotypes. (a) Key to strain placement. Indicated strains were grown at $37^{\circ}$ for 2-3 days on the following media: (b) complete medium, (c) complete medium plus $0.001 \%$ acriflavine, (d) $1 \% \mathrm{D}$-glucose plus $10 \mathrm{~mm}$ ammonium L-tartrate, (e) 1\% D-glucose plus $10 \mathrm{~mm}$ ammonium L-tartrate plus $33 \mathrm{~mm}$ molybdate, (f) $1 \%$ D-glucose plus $10 \mathrm{~mm}$ ammonium L-tartrate plus $10 \mathrm{~mm}$ allyl alcohol, (g) 1\% D-maltose plus $10 \mathrm{~mm}$ ammonium L-tartrate, (h) $50 \mathrm{~mm}$ (D)-quinate plus $10 \mathrm{~mm}$ ammonium L-tartrate, and (i) $1 \%$ D-glucose plus 10 mм 2-pyrrolidinone.

Molecular cloning of acrB: We took advantage of the close genetic linkage of $a c r B$ and $c r e B$ (CLutterbuck 1997) to clone the $a c r B$ gene. The bacterial artificial chromosome (BAC) clone that contains creB, BAC4B1 (Lockington and Kelly 2001), was tested for complementation of the acrB2 mutation in a transformation assay testing for the reversal of the resistance to acriflavine in complete medium conferred by the acrB2 mutation. This BAC clone was found to complement the acrB2 phenotype. Cosmid clones have been ordered within this BAC (Lockington and Kelly 2001) and the cosmids containing the $\mathrm{creB}$ gene, SL10A08 and RW18E04 (Lockington and Kelly 2001), were tested and failed to complement the acrB2 mutation in the transformation assay. The cosmids flanking these cosmids, SL27D09 and SL19A07, which also hybridized to BAC4B1 (Lockington and Kelly 2001), were also 
CTTGACAGTAGCTGCCTGTAATGTCAGGCCATAAAGTTTCTGCCTTAGGCAATTTTGAGGATGTTTCCAATCTTTAGGTCGTTTC GTCTTTTTCGAACCAGGTCGTCTCTTCCTCTCCTTCTTCCCGCCTCTACACT CCCCCACGCCACGTCTTATCCCCTTCACTCTCCCC

CTCTATCTTATTATTCGAATTCTTCCCCTCITIGCTCTGTGTATTCTCATATCTACTTGTTTCTTACGTTTCTCCCTTTTTGTCATT CATTCATTTTAACT GCAGAGACCCTGGCCTCGAATCCAGTCTTTTCCAAACTCCCTTCCGCCCTTTCCCGCGAACTTTTTTTTGGAAC TGCCATCGACTTCATTTCCACCTGGTCCAAGTCCTTGGCTGCTATCCGCCAAGCCGACTCGGACCTGGGTGTTGGGTTCCCTCGTCC ATGCCACGTTCATCGGCTACGGCTAGGAAGAGTCATAGCAATCGACAAGACCATGGCGGCGGCGTTCTGGGAAGAAGCCAAGCAAA $\begin{array}{lllllllllllllllllllllllllllll}M & P & R & S & S & A & T & A & R & K & S & H & S & N & R & Q & D & H & G & G & G & G & S & G & K & K & P & S & K\end{array}$ CAAAAGTCAAGTGGGCATTTAAACGCAACATACAACGGTACTGCTGGATCCGAAACTGGACCGTCTTCGCAGGTCGACTGGCCATCG $\begin{array}{lllllllllllllllllllllllllllll}Q & K & S & S & G & H & L & N & A & T & Y & N & G & T & A & G & S & E & T & G & P & S & S & Q & V & D & W & P & S\end{array}$

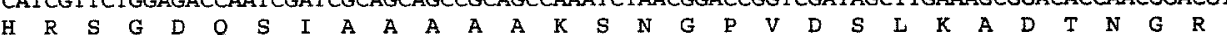
GGTTATCCGGGCGGATATGCGAAGGGAAATGCAGACATGTCTTACGGGCAGACGAATGGTGGCGTGTCGCCGAATGGTGGACTCGCC

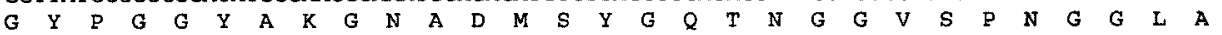
GGGCCGGCTTCACGTCGTACGGATAAGTCGGTCACTGGGACCAAGAGGACAACTTCGAATGCGTCGGTGAATCCGTTCCAGCTGGCA

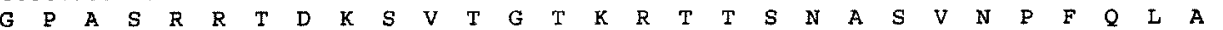
TCCACCATTCTCCGATCGTGTCCAATGTACGACACTATCGCCATCTTGATCTTTCTGCTTCAGCTCCCGCCTATGGTTCTCACTTTG

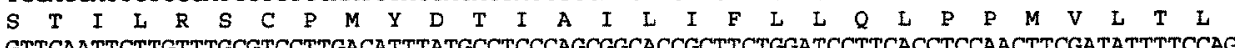

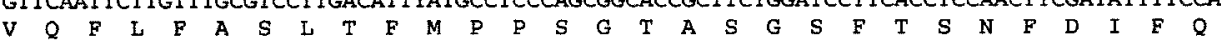
$(T+T)=\operatorname{acrB1} 4$

GGACCCGCCGGAACCCCCTCGCTCGGTACCATGATTGCAATGGATGGTTTCTGCCTGCTTGTATGGGGCCTCTTTATGTGGACGTGG $\begin{array}{llllllllllllllllllllllllllllllll}G & P & A & G & T & P & S & L & G & T & M & I & A & M & D & G & E & C & L & L & V & W & G & L & F & M & W & T & W\end{array}$ GCCCAGAATTTTGCTCTCGATTTAGCCCATGTCCAGGTTGCCATCACTCTGGGCGGTGGAGGTGCAGGGAAAAATGGTGGTGTCAAT

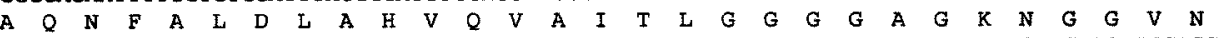
GCGCTCTGCGTCGGTATTGTTCTGATTCTGCATCTCATACGCAGCAAAGGAATACAGGATTTTGTCGTCGGCCATCTTGTTTCAGCA $\begin{array}{llllllllllllllllllllllllllllll}A & L & C & V & G & I & V & L & I & L & H & L & I & R & S & K & G & I & Q & D & F & V & V & G & H & I & V & S & A\end{array}$ AAAATCATTAGCCCCGATTTACTGTCGCATTATTCTTACCTCATGCCCGCCGAATTCAAGCGCACCGAATCGCAATCATCCCCGAGT $\begin{array}{llllllllllllllllllllllllllllllll}K & I & I & S & P & D & L & L & S & H & Y & S & Y & L & M & \text { P } & A & E & F & K & R & T & E & S & Q & S & S & P & S\end{array}$ TGGATCCGGAGCCTGCTTGCTGTACATATTCTGGCCCAGGCGGGTACTGCGATGGCGAGGCGATCGATGACTAAAAATAGGACCCCG

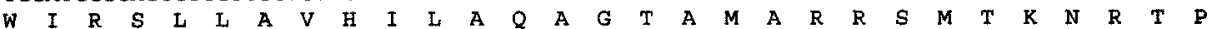
GCCCCATCACGATCAGGCAAACGCGTGGATACAGAAGCGTCTGCCGGCTCACAAACCCAGATCGACTCGGCGTTCGAATCCGCGGCC

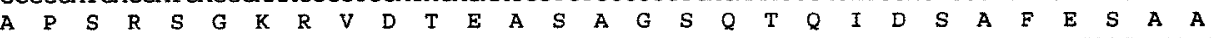
AGCGTTTCTTCCTATCTAGGCCCCGACGGGCAGATTATCACTGCCGCGCATAAGGACGGCAGGGATCGTTTGATATCGGCAAAAAAA $\begin{array}{llllllllllllllllllllllllllllllll}S & V & S & S & Y & L & G & P & D & G & Q & I & I & T & A & A & H & K & D & G & R & D & R & L & I & S & A & K & K\end{array}$ CGACGAAGGCAGGCAAATCAAGTCAGGAGCCGGCAACCTTTTTGGGCTGCACTGGCAAGCACAAAAGTCACGGTCATGAGGGAGTAT $\begin{array}{llllllllllllllllllllllllllllllllllll}R & R & R & Q & A & N & Q & V & R & S & R & Q & P & F & W & A & A & L & A & S & T & K & V & T & V & M & R & E & Y\end{array}$ GAACATTCTAGGGCCTTGTCAAAAACTGCTAGAGGACTTGCTACGACGGAGGACGATCTTCAAGGCGTTTCTTTGGACGATGGACTT

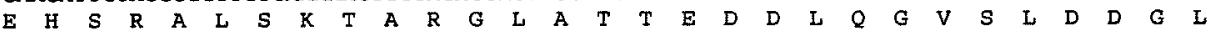
GTTTGGATTACGTATGTGGATAGCTCGACGATTAAGTTTGCAGCTGGGGATTTTGCGTCTTCGGACGACCATTCCGCGTCAGGTGTC

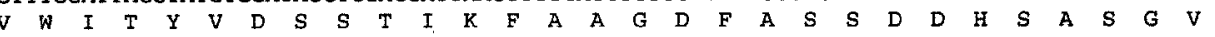
TGCGAAGCAGGCCGTGTGAGCAGCGAGGATGCGGAGCCGTTTTACGTCTGCGTCAATGGTGCGCCATGGGCAACGGTGGTCATCACT

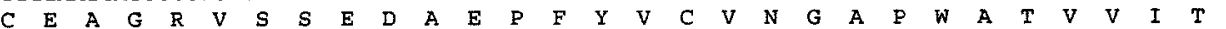
AAAGAGCATGATCCTTCAAAAGCGTCTAATACAATCTATTGGCGAGGCGAGATATCAGGTCTTGCACCCAATTGCGCGTATACTTGC $\begin{array}{llllllllllllllllllllllllllllllll}K & E & H & D & P & S & K & A & S & N & T & I & Y & W & R & G & E & I & S & G & L & A & P & N & C & A & Y & T & C\end{array}$ (.) $=\operatorname{acr} B 2$ TCTTTTGTTAAATGCGATACGGATGAGGAAATCTGCGCCATGAGTGTCAAGACCCCTGCGGCCAATGATGCAGAACAAGgtaagata $\begin{array}{lllllllllllllllllllllllllll}S & F & V & K & C & D & T & D & E & E & I & C & A & M & S & V & K & T & P & A & A & N & D & A & E & Q & A\end{array}$

(.) $=\operatorname{acrB} 15$ ttcgttctatggtttccgattcgtctcgatgtggtctaatttgtgccttccatagCCAATTCGGTGCCGGCCCCTCCGCAACCCTCA N S V P A P P Q P S TATCGACCATCCTCCCCAACAACCACGCTGAAGAACTCGATCATCAATGCTGAGGCGAAACTGAACGAAAAGCGTGCTCGACTCCGA

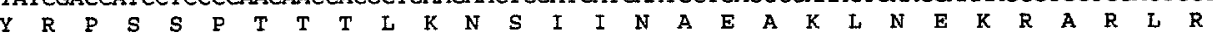
AAGGCCAAAAATGACCACAAGCTTGCTATTTCTAAGATCAAGAAGGAGCTGGACAATTACACCAATCGTCTTCAGAGCGGCACGGAT $\begin{array}{lllllllllllllllllllllllllllllll}K & A & K & N & D & H & K & L & A & I & S & K & I & K & K & E & L & D & N & Y & T & N & R & L & Q & S & G & T & D & \end{array}$ GAAAACAGGCAGAAGCAACGCTCTCTTCAATTGGAAAGGAACATTCGACAAACTGAAGAGGCTACCGCCGCTCTGGACAACCAGATC

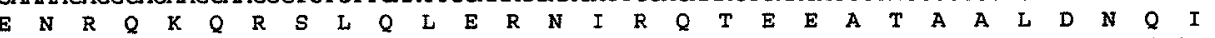
GATAACTTGGGTAATGTTCCTGACGATGAGTATCAGGAGTGGGTTGAACAGAAGGCAAAGTACGAACGTGAATTGGAGCTCCTCAAA

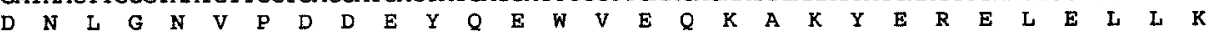
TCCGCCAAGGCAGAGATTGCTGCCACGCGTACCGCCAATGCTCGCGAGTTATCTTCATTGGAATCCGAGTTGAACTCTACCACGCAA

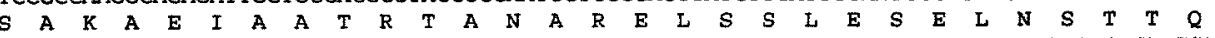
CGGCGCGAACGTCTGCAGGGTCGCCGAACCAGAGTGAATGAGCAGTACGAACGGATCATCTCGGCCAACGCACAGGGTCTCAATGAG $\begin{array}{lllllllllllllllllllllllllllll}\mathbf{R} & \mathbf{R} & \mathbf{E} & \mathbf{R} & \mathbf{L} & \mathbf{Q} & \mathbf{G} & \mathbf{R} & \mathbf{R} & \mathbf{T} & \mathbf{R} & \mathbf{V} & \mathbf{N} & \mathbf{E} & \mathbf{Q} & \mathrm{Y} & \mathbf{E} & \mathbf{R} & \mathrm{I} & \mathbf{I} & \mathbf{S} & \mathbf{A} & \mathbf{N} & \mathbf{A} & \mathbf{Q} & \mathbf{G} & \mathbf{L} & \mathbf{N} & \mathbf{E}\end{array}$ CGAGAGCGCCGCGCTGCAGAGCAGTTTGCCCGGGAACAAGATCAGTCGAAGTTGGAGCAAAGTTTCAACGAACAATTCGCGAGCATC

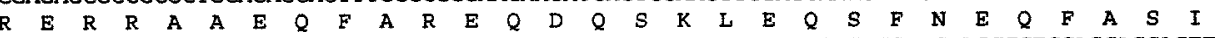
AGTCAATCAGTGCAGGATTATCAGCTGCGCACCAGTCAATTGTGGCAACAGTGTACCGCCGTCGAACAAGCCCTCCAGCAGCAGTTG

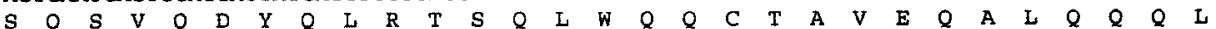
CTCATGGAGCCCGCTCCGCTAACACCCGAAGGCGAGCTGCCCGGTACTAGTACGTTTGCCGACGCGCCCAGCGTGCCCTTGGGCACA $\begin{array}{lllllllllllllllllllllllllllllllllll}L & M & E & P & A & P & L & T & P & E & G & E & L & P & G & T & S & T & F & A & D & A & P & S & V & P & L & G & T\end{array}$ TTGGCGTCAAATATGCCAAGCCACCGCTCGCTACTAGGACAGAGCTTTCCGCCGCTCAAGTCTAGTCCTCTGCAGCACTATGCTTCG

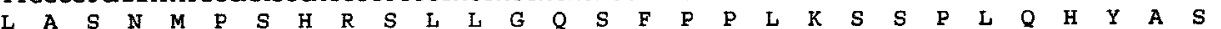
CCAATTGGAACTGCTCCGTCTCATCCGACTAGTCCAATCGCCGCTCCATCCTACCAGCCTTTCTCCAGCTCGCCATTTGGTAACGCG

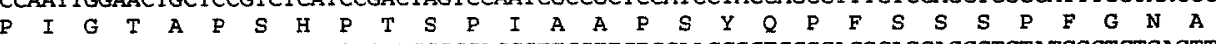
GCATCCTTCCTTGACCCGGACTTTGTCTACCGCGACCGTTCGTTCTCCAACCGCTCCGCACGCAGCAGCCTCTATGGCTCTGAGTTC $\begin{array}{lllllllllllllllllllllllllllllll}\text { A } & S & F & L & D & \text { P } & D & F & V & Y & R & D & R & S & F & S & N & R & S & A & R & S & S & L & Y & G & S & E & F\end{array}$ CCGGACGCGATAACGGCCCGCCGTGTCCCCTTTGGCGTTGATCCTTTCGAGCTCGGTAACGAGAAACGACGCGGTTCGGGATCTGAC

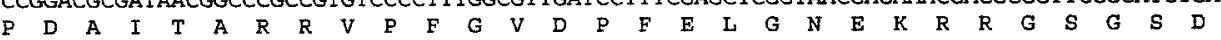
AGCACCCCGCTCAACGGTCCATCTGGCCTACGTCCTATCTCCAGTCCTTTCCAGCGAGCCGCCAGTCGCGCAAGTGGAACCGGCAGT $\begin{array}{llllllllllllllllllllllllllllllll}S & T & P & L & N & G & P & S & G & L & R & P & I & S & S & P & F & Q & R & A & A & S & R & A & S & G & T & G & S\end{array}$ GGCGGAAGCGGAGGAAGTGGTAGTGGTAGTGGCAGTCCTAGCTCTGCCCGTGGAAAGGGAAACTAAACGGAACTGCTTGCCAGCCAG $G \quad G \quad S \quad G \quad G \quad S \quad G \quad S \quad G \quad S \quad G \quad S \quad P \quad S \quad S \quad A \quad R \quad G \quad K \quad G \quad N$ * CCAACTCAGCCGTCTGCAGTGACGACAAAAGCATTGGAGATTGATCCTTACTCGCCTGCCCATATGTCCATATTTGGTCCTCGGACA TGGCTCGGACTGTCCGTGTAGTGACTGGTTATATAGTTTGCCGTCGCCAACGGTCTATAGTATAGATCCTTGTGCTCGCCGCGTAGA GCCACATCTTATATTTTGGCGAAGAAAAAGAG 
tested in a transformation assay, but again neither complemented the acrB2 mutation. Other cosmids within the region that also hybridized to BAC4B1, SW21D03, SW22F07, SW23H02, and SW23H06, were tested, and SW22F07, SW23H02, and SW23H06 were found to complement the acrB2 mutation. Thus the order of genes in this region is, from centromeric to telomeric, $a d C a d D$ acoB-atrC-acrB-creB with $a c r B \sim 30 \mathrm{~kb}$ from $\mathrm{creB}$.

Cosmid SW23H06 was selected for further analysis. An EcoRI cutdown that contained a 10-kb insert, c24F614 , complemented acrB2 in the transformation assay. c24F6-16 contained three ClaI fragments, which were cloned into pCB1004 (p4AcrB, p7AcrB, and p10AcrB), and $\mathrm{p} 4 \mathrm{AcrB}$, containing a 6 -kb insert, was found to complement the acrB2 mutation, while p7AcrB and p10AcrB did not. Plasmids containing $\sim 2$-kb $S a c$ I or $K p n I$ deletions of each end of $\mathrm{p} 4 \mathrm{AcrB}$ (p4AcrBSacI $\Delta$ and p4AcrB$\mathrm{KpnI} \Delta$ ) failed to complement acrB2 in the transformation assay. Thus $a c r B 2$ was located in the middle of the 6-kb insert of $\mathrm{p} 4 \mathrm{AcrB}$.

Sequencing of the insert in p4AcrB revealed a 3111-bp open reading frame (GenBank accession no. AF485329; Figure 3). The acrB gene contained a single intron, which was determined by sequence analysis of PCR amplified cDNAs (see MATERIALS AND METHODS), and the intronic sequence conforms to the consensus $5^{\prime}$ and $3^{\prime}$ junction and lariat sequences for fungal introns (GURR et al. 1987). To determine the transcription start point (tsp) of the gene, 5' RACE of RNA purified from D-glucose-grown mycelium was carried out using nested primers. Direct sequencing of the nested PCR product and of several subclones of this product mapped a single major tsp $-221 \mathrm{bp}$ from the translation start (Figure 3 ). A TATA-like sequence is located $33 \mathrm{bp}$ upstream of the tsp, and a CCAAT box is located 155 bp upstream of the tsp. The acrB gene is not present in the A. nidulans expressed sequence tag (EST) database (containing EST information for cDNA clones from a mixed vegetative and 24-hr asexual development culture of $A$. nidulans strains FGSCA26 constructed in lambda Zap by R. Aramayo at Texas A\&M University; http://www.genome. ou.edu/asperg.html), indicating a low level of expression under these conditions. The results of a Northern analysis using $a c r B$ as a probe were consistent with this, and $a c r B$ mRNA was below the level of detection in total RNA extracted from glucose-grown wild-type mycelia, but was present in total RNA extracted from either maltose- or fructose-grown mycelia (data not shown). This expression pattern indicates transcriptional control in response to glucose, although only a single theoretical CreA binding site is present in the 312 bases of sequence determined $5^{\prime}$ to the major start point of transcription.

The acrB gene encodes a 1015-amino-acid polypeptide. AcrB is predicted to contain three transmembrane domains near the $\mathrm{N}$ terminus, at amino acid residues 158-180 (I), 214-236 (II), and 257-274 (III), and a coiled-coil region, at amino acid residues 596-753 (SMART protein motif analysis program; SCHULTz et al. 1998). The transmembrane domain prediction program, TMHMM2 (KroGH et al. 2001), predicts the most probable location and orientation of transmembrane helices from sequence data, and this program indicated that the $\mathrm{N}$ terminus of the AcrB protein and the region between the second and third transmembrane domains are most likely cytoplasmic, with the C-terminal region of the protein predicted to be external.

A hypothetical protein is in contig 1168 of the TIGR Aspergillus fumigatus Genome Database, which shows $76 \%$ identity with the A. nidulans sequence, but these are very closely related ascomycete species. Database searches (SwissProt/SpTrEMBL/PDB) using the BlastP program (Altschul et al. 1990) revealed that the AcrB protein was not highly similar to any protein in these databases. AcrB shows most sequence similarity with hypothetical proteins, one from Neurospora crassa, Q9C2R3 (31\% identity over 1015 amino acids), and one from Saccharomyces cerevisiae, YG2K (24\% identity over 861 amino acids). The hypothetical $N$. crassa protein contains three putative transmembrane domains and a coiled-coil domain, and although it shows low similarity along the length of the protein with AcrB, specific regions such as the transmembrane domains share higher sequence identity (55\% identity over 24 amino acids). The $S$. cerevisiae hypothetical protein has a protein structure similar to that of AcrB, with three transmembrane domains and a coiled-coil region, but the sequence similarity is uniformly low throughout the protein.

Molecular analysis of the $a c r B$ mutant alleles: Three mutant alleles of $a c r B$ were sequenced to identify functional regions of the AcrB protein and to show that $a c r B$ rather than a suppressor of $a c r B 2$ had been isolated. We used a PCR approach using primers spanning the $a c r B$

Figure 3.-Location of the $a c r B$ mutations within the $a c r B$ gene. The complete sequence of the $a c r B$ genomic region is shown (GenBank accession no. AF485329). The single intron of $63 \mathrm{bp}$ is depicted in lowercase letters and the $5^{\prime}$ and $3^{\prime}$ splice junction and lariat sequences are underlined. A putative TATA box and a putative CCAAT box are underlined and in boldface letters. The major start point of transcription is indicated above the DNA by the \# symbol. A single theoretical CreA binding site in the $5^{\prime}$ untranslated region is double underlined, and a single theoretical AreA binding site in the $5^{\prime}$ untranslated region is underlined with a dashed line. The amino acid sequence is shown in single letter code beneath the DNA sequence, with numbered amino residues indicated on the left-hand side and numbered nucleotide positions indicated on the right-hand side, with the first nucleotide of the start codon as +1 . The position and nature of the DNA sequence changes in the $a c r B 2$, acrB14, and $a c r B 15$ alleles are shown, with (_) indicating a deletion of the base directly below and (+) indicating an addition of a base at the position below. The stop codon is indicated by an asterisk. 
gene followed by direct sequencing of the PCR products (see materials AND METHODS). The acrB2 mutation is a single base pair deletion at nucleotide $(n t)+1734$ at the $5^{\prime}$ splice site of the only intron (Figure 3). This would be predicted to disrupt the splicing of the intron, resulting in a frameshift after amino acid 577 and the additional residues, GRYSFYGFRFVSMWSNLCLP, before terminating. The acrB15 mutant allele contains a 2-bp deletion at $\mathrm{nt}+1815$, which results in a frameshift after amino acid 584, with an addition amino acid sequence of ATLISTILPNNHAEELDHQC before truncating. Both acrB2 and acrB15 gene products contain all three transmembrane domains but lack the coiled-coil region. The acrB14 allele contains a C-to-T transition plus a 1-bp insertion $(\mathrm{T})$ at $\mathrm{nt}+627$ that results in a frameshift after amino acid 209 and truncates 84 amino acids later. The additional amino acids are FARYHD CNGWFLPACMGPLYVDVGPEFCSRFSPCPGCHHSGR WRCREKWWCQCALRRYCSDSASHTQQRNTGFCRRP SCFSKNH. The acrB14 mutant gene product lacks two of the three transmembrane domains and the coiledcoil region and, since it is also recessive to the wild-type allele, probably represents a null allele.

\section{DISCUSSION}

In the multicellular eukaryote, A. nidulans, CreA, encoded by the $c r e A$ gene, is the master DNA-binding repressor required for carbon catabolite repression of a wide range of genes (ARsT and Cove 1973; BAILEY and Arst 1975; Arst and Bailey 1977; Hynes and Kelly 1977; Lockington et al. 1985; Dowzer and Kelly 1989, 1991; Kulmberg et al. 1993; Cubero and Scazzocchio 1994; Mathieu and Felenbok 1994; Scazzocchio et al. 1995; Shroff et al. 1996, 1997; PANOzzo et al. 1998; Mathieu et al. 2000). The creB and $\mathrm{cre} C$ genes were identified in some of the same genetic screens that identified mutations in $\mathrm{creA}$, and it is clear from the phenotypes of creB and creC mutant strains that $\mathrm{CreB}$ and $\mathrm{CreC}$ play roles under both carbon catabolite repressing and carbon catabolite derepressing conditions. Compared with creA mutations, the $\mathrm{creB}$ and $\mathrm{cre} C$ mutations lead to derepression of a restricted range of genes in the presence of a source of carbon catabolite repression such as glucose. However, compared with creA mutations, the $c r e B$ and $c r e C$ mutations have more marked effects on the expression of genes for the utilization of alternative sole carbon sources in the absence of a source of carbon catabolite repression (Hynes and Kelly 1977; Kelly and Hynes 1977; Kelly 1980; Arst 1981; Todd et al. 2000; Lockington and Kelly 2001). The $c r e B$ gene encodes a deubiquitinating enzyme (LockINGTON and KeLly 2001) and the creC gene encodes a protein that contains five WD40-repeat motifs and a proline-rich region (Todo et al. 2000), and $\mathrm{CreB}$ and $\mathrm{CreC}$ are present in a complex in vivo under both carbon catabolite repressing and derepressing conditions
(LOckIngton and Kelly 2002). What remains unclear is whether the $\mathrm{CreB} / \mathrm{CreC}$ regulatory deubiquitination network acts directly on CreA, affecting its stability, or whether it exerts its effects on carbon metabolism independently of CreA. A model has been put forward that is compatible with the existing data in A. nidulans in which a regulatory deubiquitination network involving the $\mathrm{CreB} / \mathrm{CreC}$ complex acts directly on CreA, along with other target proteins, such that the effect of $\mathrm{CreB}$ and $\mathrm{CreC}$ on carbon catabolite repression is exerted directly via an effect on the stability of CreA (LockingTON and KeLLY 2002). This model is supported by experiments showing that protein modification and/or stability of CreA could be an important component of the carbon catabolite repression mechanism (STRAuss et al. 1999). Alternatively, a model can be put forward in which the direct action of the $\mathrm{CreB} / \mathrm{CreC}$ regulatory deubiquitination complex is on permeases, transporters, or other membrane proteins, with the effects on carbon catabolite repression being the consequence of altered concentrations or cellular localizations of signaling molecules or stabilization of membrane proteins involved in glucose sensing. There are parallels with this in yeast. Some yeast permeases, including Gap1p (general amino acid permease), are subject to ubiquitindependent regulation in response to nitrogen sources (GAlan et al. 1996; Springael and Andre 1998; Rotin et al. 2000). The addition of ammonium to the medium leads to ubiquitination of Gap1, which is required for degradation of the permease. Proteins required for this process include the ubiquitin ligase, Npilp (SPRINGAEL and ANDre 1998), and Bullp and Bul2p, which form a complex with Npilp (Yashiroda et al. 1996, 1998). Brolp, which is also involved in the glucose-induced degradation of hexose transporters Hxt6/7 (SPRINGAEL et al. 2002), and Npr1p, a protein kinase that prevents Gap1 from internalization and proteolysis (DE CRAENE et al. 2001; Soetens et al. 2001), are also involved in this regulatory process. The ubiquitin hydrolase, Npi2p, is required to maintain the intracellular pool of ubiquitin (Swaminathan et al. 1999). It is possible that the CreB/ CreC complex is required to stabilize permeases and transporters by the removal of ubiquitin and in this way affect intracellular concentrations or locations of glucose or other effector molecules, or it may stabilize membrane proteins involved in glucose sensing. Although a direct or indirect effect of $\mathrm{CreB} / \mathrm{CreC}$ on CreA is discussed above as alternative models, in fact, both models could apply as they do not conflict with each other. To further characterize the roles of $\mathrm{CreB}$ and $\mathrm{CreC}$ in the cell, we have taken the approach of analyzing phenotypic suppressors of some or all of the phenotypes of $\mathrm{creB}$ and $\mathrm{cre} C \mathrm{~m}$ mutant alleles, to identify proteins involved in the ubiquitination of substrates that are deubiquinated by the CreB/CreC complex.

Phenotypic analysis of the effects of three $a \mathrm{cr} B$ alleles has shown that mutations in $a c r B$ have a broader range 
of phenotypes than the resistance to toxic compounds such as acriflavine, crystal violet, and malachite green previously described (ROPER and KAFER 1957), as they grow poorly on a range of sole carbon sources including sugars, starch, and ethanol, and they also grow poorly on some $\omega$-amino acids such as $\beta$-alanine, GABA, and pyrrolidinone either as sole carbon and nitrogen sources or as nitrogen sources in the presence of D-glucose as a carbon source. Genetic analysis has shown that the $a c r B 2$ mutation is a suppressor of the phenotypes conferred by mutations in $\mathrm{creB}$ and $\mathrm{cre} C$. Strains containing the creB1937 and creC27 mutations show derepressed expression of the alcA gene, in that there is inappropriate expression in the presence of D-glucose, and the presence of the acrB2 mutation completely reverses the effects of these mutations as judged by plate testing. The reduced growth of the acrB2-containing strain on medium containing ethanol as a sole carbon source may indicate that this is due to a failure to induce alc $A$ under either repressing or derepressing conditions due to the loss of $a c r B$ and that this failure in induction overrides the derepression caused by $\mathrm{cre} B$ and $\mathrm{cre} C \mathrm{mu}$ tations. The $\mathrm{creB} 1937$ and $\mathrm{creC} 27$ mutations lead to poor growth on D-quinate and D-glucuronate as sole carbon sources, and the acrB2 mutation partially repairs this defect. These phenotypes are consistent with a regulatory defect associated with the membrane and signaling, but not with a simple interpretation that only permeases and transporters are affected, as in the case of ethanol, a fat soluble compound, no permease is required or exists.

The amino acid sequence of AcrB indicates that the protein contains membrane-spanning domains, indicating the probability of its residing in the membrane. There is also a coiled-coil region that would allow the prediction that the protein forms either a homodimer or a heterodimer. The striking aspect of the sequence is the lack of sequence similarity with proteins in databases from other eukaryotes, including those for which the entire genome has been sequenced, and it is clearly not a direct homolog of any gene in a characterized signaling pathway. If functional homologs exist they are very diverged in sequence from $\mathrm{AcrB}$ and thus unrecognizable. The CreB and CreC proteins are involved in a deubiquitination network that removes ubiquitin from target proteins, and although these target proteins are not yet directly identified, the mutant phenotypes indicate that they are proteins involved in carbon metabolism and its regulation. Unlike AcrB, CreB and CreC are well conserved among most eukaryotes other than yeast. The acrB2 mutation suppresses phenotypes of the cre $B$ and $\operatorname{cre} C$ mutations and this suggests that $A c r B$ is involved in a process opposite to deubiquitination, such as a ubiquitin ligase pathway, since a failure to add ubiquitin to substrates could suppress the phenotypic effects of mutations that affect the removal of ubiquitin moieties. Thus the ubiquitination/deubiquitination net- work in A. nidulans that involves $\mathrm{CreB}, \mathrm{CreC}$, and $\mathrm{AcrB}$ provides an ideal genetic and molecular genetic system in which to unravel regulation of protein stability.

We thank H. N. Arst for generously providing strains containing acrB14 and $a c r B 15$. This work was supported by an Australian Research Council grant to J.M.K. and an Australian Postgraduate Research Award to N.A.B.

\section{LITERATURE CITED}

Altschul, S. F., W. Gish, W. Miller, E. W. Myers and D. J. Lipman, 1990 Basic local alignment search tool. J. Mol. Biol. 215: 403410.

ARst, H. N., 1981 Aspects of the control of gene expression in fungi. Symp. Soc. Gen. Microbiol. 31: 131-160.

Arst, H. N., and C. R. Bailey, 1977 The regulation of carbon metabolism in Aspergillus nidulans, pp. 131-146 in Genetics and Physiology of Aspergillus nidulans, edited by J. E. Sмiтн and J. A. Pateman. Academic Press, London.

Arst, H. N., and D. J. Cove, 1973 Nitrogen metabolite repression in Aspergillus nidulans. Mol. Gen. Genet. 126: 111-141.

Bailey, C. R., and H. N. ARst, 1975 Carbon catabolite repression in Aspergillus nidulans. Eur. J. Biochem. 51: 573-577.

Brody, H., J. Griffith, A. J. Cuticchia, J. Arnold and W. E. TimBERLAKE, 1991 Chromosome-specific recombinant DNA libraries from the fungus Aspergillus nidulans. Nucleic Acids Res. 19: 3105-3109.

Cadavid, A. L. M., A. Ginzel and J. A Fischer, 2000 The function of the Drosophila Fat facets deubiquitinating enzyme in limiting photoreceptor cell number is intimately associated with endocytosis. Development 127: 1727-1736.

Carroll, A. M., J. A. Sweigard and B. Valent, 1994 Improved vectors for selecting resistance to hygromycin. Fungal Genet. Newsl. 41: 22.

Chen, X., and J. A. Fischer, 2000 In vivo structure/function analysis of the Drosophila fat facets deubiquitinating enzyme gene. Genetics 156: 1829-1836.

Chen, X., B. Zhang and J. A. Fischer, 2002 A specific protein substrate for a deubiquitinating enzyme: Liquid facets is the substrate of Fat facets. Genes Dev. 16: 289-294.

Clutterbuck, A. J., 1974 Aspergillus nidulans genetics, pp. 447-510 in Handbook of Genetics, edited by R. C. King. Plenum, New York.

Clutterbuck, A. J., 1993 Fungi: A. nidulans (nuclear genes), pp. 3.71-3.84 in Genetic Maps: Locus Maps of Complex Genomes, edited by S. J. O'Brien. Cold Spring Harbor Laboratory Press, Cold Spring Harbor, NY.

Clutterbuck, A. J., 1997 The validity of the Aspergillus nidulans linkage map. Fungal Genet. Biol. 21: 267-277.

Cove, D. J., 1966 The induction and repression of nitrate reductase in the fungus Aspergillus nidulans. Biochim. Biophys. Acta 133: $51-56$.

Cubero, B., and C. Scazzocchio, 1994 Two different, adjacent and divergent zinc finger binding sites are necessary for CreA mediated carbon catabolite repression in the proline gene cluster of Aspergillus nidulans. EMBO J. 13: 407-415.

De Craene, J.-O., O. Soetens and B. Andre, 2001 The Npr1 kinase controls biosynthetic and endocytic sorting of the Yeast Gap1 permease. J. Biol. Chem. 276: 43939-43948.

Dowzer, C. E. A., and J. M. Kelly, 1989 Cloning of creA from Aspergillus nidulans: a gene involved in carbon catabolite repression. Curr. Genet. 15: 457-459.

Dowzer, C. E. A., and J. M. Kelly, 1991 Analysis of the creA gene, a regulator of carbon catabolite repression in Aspergillus nidulans. Mol. Cell. Biol. 11: 5701-5709.

Galan, J. M., V. Moreau, B. Andre, C. Volland and R. HaguenauerTsaPIS, 1996 Ubiquitination mediated by the Npi1/Rsp5p Ubiquitin-protein ligase is required for endocytosis of the yeast uracil permease. J. Biol. Chem. 271: 10946-10952.

GurR, S. J., S. E. UnkLES and J. R. Kinghorn, 1987 The structure and organisation of nuclear genes of filamentous fungi, pp. 93-139 in Gene Structure in Eukaryotic Microbes, edited by J. R. Kinghorn. IRL Press, Oxford. 
Hynes, M. J., and J. A. Pateman, 1970 The genetic analysis of regulation of amidase synthesis in Aspergillus nidulans. I. Mutants able to use acrylamide. Mol. Gen. Genet. 108: 95-106.

Hynes, M.J., and J. M. Kelly, 1977 Pleiotropic mutants of Aspergillus nidulans altered in carbon metabolism. Mol. Gen. Genet. 150: 193-204.

KeLly, J. M., 1980 Pleiotropic mutants of Aspergillus nidulans affected in carbon metabolism. Ph.D. Thesis, La Trobe University, Melbourne.

Kelly, J. M., and M.J. Hynes, 1977 Increased and decreased sensitivity to carbon catabolite repression of enzymes of acetate metabolism in mutants of Aspergillus nidulans. Mol. Gen. Genet. 156: 87-92.

Krogh, A., B. Larsson, G. von Hijne and E. L. L. Sonnhammer, 2001 Predicting transmembrane protein topology with a hidden Markov model: application to complete genomes. J. Mol. Biol. 305: $567-580$.

Kulmberg, P., M. Mathieu, C. E. A. Dowzer, J. M. Kelly and B. Felenbok, 1993 Specific binding sites in the alcR and alcA promoters of the ethanol regulon for the CreA repressor mediating carbon catabolite repression in Aspergillus nidulans. Mol. Microbiol. 7: 847-857.

LeE, S. B., and J. W. TAYLOR, 1990 Isolation of DNA from fungal mycelia and single spores, pp. 282-287 in PCR Protocols: A Guide to Methods and Applications, edited by M. A. InNis, D. H. GeLfand, J. S. Sninsky and T. J. White. Academic Press, London.

Lockington, R. A., and J. M. KeLly, 2001 Carbon catabolite repression in Aspergillus nidulans involves deubiquitination. Mol. Microbiol. 40: 1311-1321.

Lockington, R. A., and J. M. Kelly, 2002 The WD40-repeat protein CreC interacts with and stabilizes the deubiquitinating enzyme CreB in vivo in Aspergillus nidulans. Mol. Microbiol. 43: 1173-1182.

Lockington, R. A., H. M. Sealy-Lewis, C. Scazzocchio and R. W. Davies, 1985 Cloning and characterization of the ethanol utilization regulon of Aspergillus nidulans. Gene 33: 137-149.

Mathieu, M., and B. Felenbok, 1994 The Aspergillus nidulans CREA protein mediates glucose repression of the ethanol regulon at various levels through competition with the ALCR-specific transactivator. EMBO J. 13: 4022-4027.

Mathieu, M., S. Fillinger and B. Felenbok, 2000 In vivo studies of upstream regulatory cis-acting elements of the alcRgene encoding the transactivator of the ethanol regulon in Aspergillus nidulans. Mol. Microbiol. 36: 123-131.

OAkley, C. E., C. F. Weil, P. L. Kretz and B. R. OAkley, 1987 Cloning of the riboB locus of Aspergillus nidulans. Gene 53: 293-298.

Oldham, C. E., R. P. Mohney, S. L. H. Miller, R. N. Hanes and J. P. O'Bryan, 2002 The ubiquitin-interacting motifs target the endocytic adaptor protein epsin for ubiquitination. Curr. Biol. 12: 1112-1116.

Panozzo, C., E. Cornillot and B. Felenbok, 1998 The CreA repressor is the sole DNA-binding protein responsible for carbon catabolite repression of the alcA gene in Aspergillus nidulans via its binding to a couple of sites. J. Biol. Chem. 273: 6367-6372.

Pateman, J. A., B. M. Rover and D. J. Cove, 1967 Genetical and biochemical studies of nitrate assimilation in Aspergillus nidulans. Biochem. J. 104: 103-111.

Roper, J. A., and E. KAFER, 1957 Acriflavine resistant mutants of Aspergillus nidulans. J. Gen. Microbiol. 16: 660-667.

Rotin, D., O. Staub and R. Haguenauer-Tsapis, 2000 Ubiquitination and endocytosis of plasma membrane proteins: role of Nedd4/Rsp5p family of ubiquitin-protein ligases. J. Membr. Biol. 176: $1-17$.

Sambrook, J., E. F. Fritsch and T. Maniatis, 1989 Molecular Cloning: A Laboratory Manual. Cold Spring Harbor Laboratory Press, Cold Spring Harbor, NY.

Scazzocchio, C., V. Gavrias, B. Cubero, C. Panozzo, M. Mathieu et al., 1995 Carbon catabolite repression in Aspergillus nidulans—a review. Can. J. Bot. 73: S160-S166.

Schultz, J., F. Milpetz, P. Bork and C. P. Ponting, 1998 SMART, a simple modular architecture research tool: identification of signaling domains. Proc. Natl. Acad. Sci. USA 95: 5857-5864.

Shroff, R. A., R. A. Lockington and J. M. Kelly, 1996 Analysis of mutations in the $\mathrm{creA}$ gene involved in carbon catabolite repression in Aspergillus nidulans. Can. J. Microbiol. 42: 950-959.

Shroff, R. A., S. M. O'Connor, M. J. Hynes, R. A. Lockington and J. M. Kelly, 1997 Null alleles of creA, the regulator of carbon catabolite repression in Aspergillus nidulans. Fungal Genet. Biol. 22: $28-38$.

Soetens, O., J.-O. De Craene and B. Andre, 2001 Ubiquitin is required for sorting to the vacuole of the yeast general amino acid permease, Gap1. J. Biol. Chem. 276: 43949-43957.

SPRINGAEL, J.-Y., and B. ANDRE, 1998 Nitrogen-regulated ubiquitination of Gap1 Permease of Saccharomyces cerevisiae. Mol. Biol. Cell 9: $1253-1263$.

Springael, J.-Y., E. Nikko, B. Andre and A. M. Marini, 2002 Yeast Npi3/Brol is involved in ubiquitin-dependent control of permease trafficking. FEBS Lett. 517: 103-109.

Strauss, J., R. L. Mach, S. Zeilinger, G. Hartler, G. Stoffler et al., 1999 Cre1, the carbon catabolite repressor protein from Trichoderma reesei. FEBS Lett. 376: 103-107.

Swaminathan, S., A. Y. TAmerik and M. Hoechstrasser, 1999 The Doa4 Deubiquitinating enzyme is required for ubiquitin homeostasis in Yeast. Mol. Biol. Cell 10: 2583-2594.

Tilburn, J., C. Scazzocchio, G. T. Taylor, J. H. Zabicky-Zissman, R. A. Lockington et al., 1983 Transformation by integration in Aspergillus nidulans. Gene 26: 205-221.

Todd, R. B., R. A. Lockington and J. M. Kelly, 2000 The Aspergillus nidulans creC gene involved in carbon catabolite repression encodes a WD40 repeat protein. Mol. Gen. Genet. 263: 561-570.

Wu, Z. R., Q. H. Li, M. E. Fortini and J. A. Fischer, 1999 Genetic analysis of the role of the Drosophila fat facets gene in the ubiquitin pathway. Dev. Genet. 25: 312-320.

Yashiroda, H., T. Oguchi, Y. Yasuda, A. Toh-E and Y. Kikuchi, 1996 Bull, a new protein that binds to the Rsp5 Ubiquitin ligase in Saccharomyces cerevisiae. Mol. Cell. Biol. 16: 3255-3263.

Yashiroda, H., T. D. Kaida, A. Toh-E, and Y. Kikuchi, 1998 The PY-motif of Bull protein is essential for growth of Saccharomyces cerevisiae under various stress conditions. Gene 225: 39-46.

Communicating editor: M. S. SACHS 